\title{
Basteln, matschen und toben während der Coronakrise
}

\author{
Die Bedeutung von wohnungsnahem Freiraum für Kinder und Familien während der \\ Frühphase der Pandemie
}

Henriette Bertram, Stefanie Hennecke, Angela Million, Johanna Niesen

\begin{abstract}
Mit der Sperrung von Spielplätzen und Parkanlagen bei zeitgleicher Schließung aller Einrichtungen der Kinderbetreuung zu Beginn der Coronapandemie wandelten sich Bedeutung und Nutzung des öffentlichen Raums. Maßnahmen und Empfehlungen wie das Abstandhalten, die Schließung von Schulen und Betreuungseinrichtungen und das Arbeiten von zu Hause veränderten den Kinder- und Familienalltag von einem Tag auf den anderen. Viele sonst alltäglich genutzte öffentliche Freiräume standen nicht mehr zur Verfügung; das „Austoben“ auf dem Spielplatz fiel ebenso weg wie Treffen mit Freund_innen oder Großeltern im Park. In unserem Artikel betrachten wir die pandemiebedingten Einschränkungen im Alltag von Kindern und Familien sowie individuelle Kompensationsstrategien: Wie haben sich die Wohn- und Freiraumpraktiken von Kindern verschoben und wie hat sich das im Raum niedergeschlagen? Was bedeutet der an die Wohnung angrenzende private und öffentliche Freiraum für die Kinder und ihre Familien? Welche Bedarfe an öffentlichen und wohnungsnahen Freiräumen ergeben sich in Zeiten der Pandemie und darüber hinaus? Mithilfe narrativer Landkarten analysieren wir Bewegungsradius, Tagesrhythmus und Freiraumpraxis von Kindern. Befragt werden Kinder zwischen acht und zwölf Jahren sowie deren Eltern in zwei kleineren Großstädten in Westdeutschland.
\end{abstract}

Ersteinreichung: 10. Dezember 2020; Veröffentlichung online: 26. November 2021 An English abstract can be found at the end of the document.

\section{Ausgangslage: Pandemiebedingte Verschiebungen in Familienalltag und Freiraumpraktik}

Mit Beginn der Coronapandemie wurden im März2020 in Deutschland und vielen anderen Ländern alleEinrichtungen der Kinderbetreuung geschlossen. Diese Schließungen von Schulen, Horten und Kindertagesstätten dauerten teilweise bis zum Beginn der Sommerferien (je nach Bundesland zwischen J uni und August) an. Nurtage oder gruppenweisekonnten Kindernach und nach in die Bildungs- und Betreuungseinrichtungen zurückkehren, zeitweise war dafür der Nachweis notwendig, dass die Eltern in „systemrelevanten“ Berufen tätig waren. Zeitgleich erfolgtein vielen Städten und Gemeinden die Sperrungvon Spielplätzen oder gar ganzen Parkanlagen. Diese Maßnahmen beeinflussten schlagartig die Wahrnehmung der Bedeutung und Nutzung des öffentlichen Freiraums.

„Social Distancing“, „Homeschooling“, „Homeoffice“ und die ausdrückliche Empfehlung, sich zu Hause aufzuhalten, veränderten den Kinder- und 
Familienalltag radikal. Viele sonst alltäglich genutzte öffentliche Freiräume standen über Nacht nicht mehr zur Verfügung; das „Austoben“ auf dem Spielplatz fiel ebenso weg wie Treffen mit Freund_innen im Park oder das Picknick mit den Großeltern. Die Wohnung wurde für längere Zeit zum Zentrum des Alltags und diente zugleich als Schule, Arbeitsplatz und Freizeitort. Die ohnehin herrschende Begrenztheit des noch verbleibenden Freiraums der Bürgersteige und Straßen wurde durch das Bemühen, Abstandsregeln einzuhalten, offensichtlich. Viel befahrene Straßen, dicht beparkte Seitenstreifen und schmale Bürgersteige zeigten die Unmöglichkeit für Fußgänger_innen und Radfahrer_innen, sich im Freiraum mit Abstand, also sicher vor Infektionen, zu bewegen. Verstärkt wurde dieser Effekt dadurch, dass viele den öffentlichen Nahverkehr mieden und alternativ dazu das Fahrrad oder das Auto für die täglichen Wege nutzten. Andererseits eröffnete das Wegfallen von Arbeitswegen mancherorts auch neue Freiräume, wenn etwa Firmenparkplätze leer standen und für Spiel und Sport angeeignet werden konnten. Da die Nutzung des öffentlichen Freiraums in dieser Weise über mehrere Wochen hin eingeschränkt war, gewann die Verfügbarkeit über wohnungsnahe Freiräume eine umso größere Bedeutung.

Diese Erfahrungen teilten alle Stadtbewohner_innen, und sie wurden in zahlreichen Zeitungsartikeln aus der Perspektive von Erwachsenen beschrieben und reflektiert. Mit unserer Untersuchung stellen wir ihnen die Perspektive von Kindern gegenüber. In unserem Artikel betrachten wir die pandemiebedingten Einschränkungen im Alltag von Kindern und Familien sowie die individuellen Kompensationsstrategien anhand einer im Oktober 2020 durchgeführten Untersuchung in zwei kleineren Großstädten in Westdeutschland: Wir fragen, wie sich die Wohn- und Freiraumpraktiken von Kindern in der Frühphase der Pandemie verschoben haben und welche Bedeutung der an die Wohnung angrenzende private und/oder öffentliche Freiraum für die Kinder und ihre Familien in dieser Phase hatte, auch im Hinblick auf unterschiedliche Lebens- und Wohnsituationen. Wir erhoffen uns Erkenntnisse in Bezug auf Bedarfe an öffentlichen und wohnungsnahen Freiräumen auch über die Zeit der Pandemie hinaus. Methodisch stützt sich der BeitragaufnarrativeLandkarten und begleitendenarrativeInterviewsmit Kindern im Grundschulalter sowieihren Eltern. Die Untersuchungentstand als Vorstudie im Rahmen eines größeren Forschungsvorhabens zur Nutzung wohnungsnaher Freiräume durch Kinder und Jugendliche an der Universität Kassel in Zusammenarbeit mit der Technischen Universität (TU) Berlin. Aufgrund der stark einschränkenden Bedingungen des zweiten Lockdowns im Herbst 2020 ist der Kreis der befragten Familien auf eine kleine Gruppe aus der sozial gut abgesicherten Mittelschicht beschränkt. Diesen Umstand reflektieren wir im Fazit unseres Beitrags.

Im Folgenden geben wir zunächst einen Überblick über den aktuellen Stand der Forschung zu den Auswirkungen der pandemiebedingten Einschränkungen auf den Alltag von Kindern undJ ugendlichen. Sodann führen wir in die Methode der narrativen Landkarten ein und beschreiben unser Vorgehen. Im Anschluss stellen wir unsere Ergebnisse nach Raumkategorien sortiert vor und diskutieren unterschiedliche Strategien der Kompensation von Einschränkungen während der Schließungszeiten. Schließlich leiten wir daraus Forschungsfragen für weitere Untersuchungen zu diesem Thema ab. 


\section{Kinder und Freiraumnutzung während der Covid-19- Pandemie: Stand der Forschung}

DieweltweiteCoronapandemiegehtmitEinschränkungen und Ängsten sowie mit gesundheitlichen Gefahren für eine Vielzahl von Menschen einher. Durch die veränderten Rahmenbedingungen des Alltags werden gesellschaftliche Themen und Tendenzen akzentuiert oder infrage gestellt. Auch für viele wissenschaftliche Disziplinen ist diese gesellschaftliche Sondersituation interessant, weshalb von Beginn an viele, entsprechend kurzfristig geplante und umgesetzte Untersuchungen entstanden, die sich mit den veränderten Bedingungen in Zeiten der Pandemie beschäftigen.

Erste Ergebnisse der Studien zum Einfluss des Lockdowns auf Familien mit Kindern ab März 2020 wurden bereits im April 2020 veröffentlicht. Die meist über das Internet durchgeführten Umfragen trafen ohne Ausnahme auf große Resonanz, sind andererseits aber - nach Aussage der Autor_innen - in ihrer Aussagekraft begrenzt, weil in der kurzen Zeit über die Abfragen keine repräsentative Stichprobe erreicht werden konnte (vgl. Andresen et al. 2020a: 7; Langmeyer et al. 2020: 2). Zudem beteiligten sich Familien in herausfordernden sozialen Konstellationen, etwa Alleinerziehende, Familien mit vier und mehr Kindern oder Familien mit Migrationshintergrund, nur in geringem Umfang.

Befragt wurden jeweils Eltern zu ihrer veränderten familiären Alltagssituation. Nahezu alle im Folgenden referierten Studien schließen mit der Forderung, die Auswirkungen des Lockdowns insbesondere für Familien in sozial prekären Verhältnissen genau zu untersuchen, und weisen darauf hin, dass eine Abwägungzwischen der Gefahr, dass Kinder Covid-19übertragen, und den negativen Auswirkungen einer langfristigen sozialen Isolation von Kindern notwendig sei (vgl. DAKJ 2020; Andresen et al. 2020b). Seit der Durchführung unserer Studie werden laufend weitere Forschungsergebnisse auf der Basis empirischer Studien publiziert, unter anderem die Stellungnahme des Bundesjugendkuratoriums (BJ K) Kindheit und J ugend in Zeiten von Corona aus dem Mai 2021(Schröer 2021) oder der Ergebnisbericht des Deutschen J ugendinstituts (DJ I) von 2021 Die Situation J ugendlicher in der Corona-Krise (Walper et al. 2021). In der Tendenz wird hier der Forschungsstand aus dem J ahr 2020 bestätigt.

Die Schließung von Betreuungseinrichtungen und Vereinen im Frühling 2020 forderte in einer Gesellschaft, die seit dem 20. J ahrhundert Kindheit zeitlich und räumlich überwiegend in institutionalisierten Kontexten organisiert (Kampmann 2004) und in der eine Scholarisierung selbst in der Freizeit ablesbar ist (Fölling-Albers 2000), gravierende Umstellungen im Alltag (Fischer et al. 2020). Eine Studie aus Thüringen fragte direkt danach, was Kinder während der Kontaktbeschränkungen vermissten. Neben Freund_innen und Spielpartner_innen (94 Prozent) sowie anderen Bezugspersonen, zu denen kein Kontakt mehr möglich sei, wurden häufig „Bewegung und im Freien sein“ (31 Prozent) genannt. Hier fand insbesondere der Zugang zum Spielplatz Erwähnung (Lochner 2020: 3 f.). Ein Literatur-Review aus England vom J uni 2020 kommt zu dem Ergebnis, dass es für Kinder eine erhebliche Beeinträchtigung darstellt, von Orten des Spielens und vom Spielen mit Gleichaltrigen dauerhaft abgehalten zu werden (vgl. Ball/Gill/Yates 2020). 
Folgt man der Literatur zur kindlichen Entwicklung, so tragen frische Luft und Bewegung essenziell zur körperlichen und geistigen Gesundheit bei (vgl. Moran/Plaut/Merom 2017). Der Zugang zu Freiraum beziehungsweise zu Grünflächen und somit die Möglichkeit von Naturerfahrung ist hierbei zentral (vgl. Gebhard 2013). Auch unabhängig vom Alter ist die zentrale gesundheitliche Wirkung von Freiräumen im Lebensalltag vielfach belegt (vgl. Claßen 2020). Entsprechend thematisieren einige jüngere Fachbeiträge mögliche gesundheitliche Konsequenzen der Schließung von öffentlichen Grünflächen (vgl. Honey-Rosés et al. 2020; Razani/Radhakrishna/ Chan 2020; Slater/Christiana/Gustat 2020) insbesondere auch für Kinder (vgl. Graber et al. 2020; Moore et al. 2020).

Eine nahe liegende Ausweichstrategie für Familien lag in der vermehrten Nutzung privater Freiflächen, soweit diese verfügbar waren. In einer für das DJI durchgeführten Studie mit 10.000 Teilnehmer_innen wird deutlich, dass 71 Prozent der Befragten zeitweise oder durchgehend Zugang zu privat nutzbaren Freiräumen wie eigenen Gärten oder Balkonen haben (vgl. Langmeyer et al. 2020: 8 f.). Die in Deutschland gegebene hohe Verfügbarkeit dieser Freiräume bestätigt auch eine Auswertung des Sozio-oekonomischen Panels (SOEP) durch das Institut der Deutschen Wirtschaft (IW). Übereinstimmende Daten des SOEP von 2017 und 2018 und des DJI belegen, dass in Deutschland rund zwei Drittel der Familien Zugang zu einem Garten haben. Sie zeigen aber gleichzeitig, dass die Verfügbarkeit sozial sehr differenziert betrachtet werden muss. Während kinderreiche Familien zu etwa 70 Prozent Zugang zum eigenen Garten haben, steht ein solcher nur weniger als 50 Prozent der Familien mit einem alleinerziehenden Elternteil, mit einem Migrationshintergrund oder mit ALG-II-Bezug zur Verfügung (vgl. Geis-Thöne 2020: 7 f.). Kinder aus Familien, denen privat nutzbarer Freiraum nicht zur Verfügung steht, sind gleichzeitig oft von Armut betroffen oder armutsgefährdet (vgl. Bertelsmann-Stiftung 2020: 4; Bähr et al. 2020). Aus diesem Grund verfügen sie zudem über wenig Ressourcen, einen Zugang zu Freiraum alternativ zu organisieren, etwa über Ausflüge, Besuche oder Reisen - Aktivitäten, die während der pandemiebedingten Schließungen ohnehin stark eingeschränkt waren. Im Jahr 2018 war jedes fünfte Kind in Deutschland - das sind 2,8 Millionen Kinder - von Armut betroffen oder armutsgefährdet (vgl. Bertelsmann-Stiftung 2020: 4). Es ist also zu vermuten, dass die in unserer Studie ermittelten Herausforderungen, die ein eingeschränkter Freiraumzugang mit sich bringt, verstärkt für sozial weniger privilegierte Familien und Kinder bestehen.

Neben privat nutzbaren Freiräumen wurden Innenräume und öffentliche Plätze gleichermaßen als wichtige Orte für veränderte Freizeitaktivitäten genannt (vgl. Langmeyer et al. 2020: 9). Die Studie des DJI zeigt ebenso wie die sogenannteKiCo-Studie(Kinder, Eltern und ihreErfahrungen während der Corona-Pandemie; Andresen et al. 2020a), dass innerfamiliäre Spannungen während der Schließungszeit stiegen und Konflikte häufiger laut ausgetragen wurden (vgl. Langmeyer et al. 2020: 18f.; Andresen et al. 2020a: 19). Insofern ist die Frage nach Möglichkeiten des Rückzugs in der eigenen Wohnung etwa in ein eigenes Zimmer oder zumindest an einen eigenen Schreibtisch - ebenso zentral wie die Frage nach einem eigenen Garten oder anderen Freiräumen, die eine Möglichkeit des Ausweichens in Konfliktsituationen bieten. 
Diese „Freiräume“ in der Wohnung stehen laut SOEP-Daten immerhin 85 Prozent aller Zwölfjährigen in Deutschland zur Verfügung, wobei auch hier die Anteile bei sozial benachteiligten Familien erwartungsgemäß geringer sind (vgl. Geis-Thöne 2020: 6).

In der Studie des DJI gaben 65 Prozent der Befragten an, in der Freizeit öffentliche Freiräume aufzusuchen, auch wenn ein eigener Garten zur Verfügung steht (vgl. Langmeyer et al. 2020: 9). Während die Nutzung der eigenen Wohnung als Freiraum nicht zwangsläufig von einer Aufsicht durch die Eltern abhängt, konnten gerade jüngere Kinder Ausflüge in öffentliche Freiräumenurin Begleitungihrer Eltern unternehmen, waren also auf deren Zeit und Bereitschaft angewiesen. Und auch hier sind der ausgeübte Beruf und damit verbunden der soziale Status limitierende Faktoren dafür, ob Ausflüge für die Eltern überhaupt möglich und mit der Erwerbsarbeit vereinbar sind. Wenn beispielsweise die Aufsicht unter mehreren Erwachsenen aufgeteilt werden konnte oder mobiles, zeitlich flexibles Arbeiten möglich war, waren entsprechend auch gemeinsame Freizeitaktivitäten leichter unterzubringen:

„Auch wenn Outdooraktivitäten, wie Wandern und Radfahren, im Familienkreis auch weiterhin möglich sind, sind die Kinder vor diesem Hintergrund in der Regel gezwungen, den größten Teil des Tages zu Hause zu verbringen. Das hat zur Folge, dass die Ressourcen, die sie hier vorfinden, derzeit fast allein darüber entscheiden, wie sie ihren Alltag gestalten und wie gut sie lernen können.“ (Geis-Thöne 2020: 3)

Die KiCo-Studie mit rund 25.00o Teilnehmer_innen verweist auf die Ambivalenz der Ergebnisse, die sowohl Defizite als auch Vorzüge der neuen Alltagssituation erkennen lassen. Eltern erlebten den Wegfall eines durchorganisierten Alltags einerseits als Entlastung, andererseits aber auch als starke Überlastung und Verunsicherung durch neue komplexe Herausforderungen:

„Das Wegbrechen der Infrastruktur für Kinder und Jugendliche und die neue Situation in den Betreuungs-, Freizeit- und Bildungsangeboten sowie die teilweise erlebte Neuordnung der Erwerbsarbeit trifft Familien unterschiedlich. Für die einen findet plötzlich alles zu Hause mit gleich gebliebenen Erwartungen und Herausforderungen statt, andere finden neue Erwartungen und gemeinsameZeit. Erfahrungen aus dieser Zeit differenziert auszuwerten ist eine Chance, um auch zukünftige Diskussionen um das Verhältnis von Eltern, Kindern und öffentlichen Betreuungs- und Bildungseinrichtungen sowie Arbeitgeber_innen neu und alltagsorientierter zu diskutieren." (Andresen et al. 2020a: $22 \mathrm{f}$.)

In der hier vorgestellten Studie betrachten wir speziell die geänderte Erfahrung der Freiraumnutzung in dieser Zeit.

Die Erkenntnisse aus den Umfragen werden von einigen Reflexionen und Thesenpapieren zur Notwendigkeit privat verfügbarer und öffentlich zugänglicher Freiräume flankiert. Das Wuppertal Institut für Klima, Umwelt, Energie verweist in seinem Diskussionspapier zu einer resilienten „PostCorona-Stadt" auf die Bedeutung von Freiräumen für die Stadt in der Krise und fordert, „Nah-Erholungsmöglichkeiten” zu stärken und die „Bedeutung von privat verfügbarem Freiraum und die damit verbundenen sozialen 
Privilegierungen" bei zukünftigen Planungen besonders zu berücksichtigen (Schneidewind et al. 2020: 7). Das Memorandum zur Post-Corona-Stadt des Bundesministeriums für Bildung und Forschung vom Juli 2020 nennt Forschungsbedarfe vor allem in den Handlungsfeldern der „qualitativen Weiterentwicklungvon Quartieren, insbesondere[...] [bezüglich des] Umgang[s] mit Zielkonflikten wie jenen zwischen baulicher Verdichtung und Freiraumentwicklung sowie [...] [der] Erschließung von (auch kleinteiligen) Flächen- und Nutzungspotenzialen“ (BMBF 2020: 6). Zugleich zeigten lokale ehrenamtliche Initiativen von Eltern in Zusammenarbeit mit der lokalen Stadtverwaltung in dicht bebauten Vierteln mit einem Mangel an Freiräumen, wie zumindest temporär Freiräume für Kinder etwa in Form von Spielstraßen zur Entlastung überfüllter Spielplätze während der Pandemie geschaffen werden konnten (Million 2021b). Es ist zu betonen, dass in der Fachliteratur bereits vor der Covid-19-Pandemie das Defizit an verfügbaren und adäquat ausgestatteten Freiräumen für Kinder insbesondere im Nahbereich des Wohnens diskutiert wurde (vgl. Spitthöver 2002). Die längst nicht mehr neuen Ergebnisse bekommen allerdings durch die Pandemie - und die Wahrscheinlichkeit weiterer ähnlicher Situationen in der Zukunft - eine neue Aktualität und Dringlichkeit.

\section{Narrative Landkarten: Methodik}

Im Rahmen der sozialräumlichen Kindheitsforschung werden die Aneignung und Nutzung von (Frei-)Räumen häufig mit Verfahren der visuellen Sozialforschung, insbesondere mit subjektiven Landkarten, untersucht (vgl. Katz 2004; von Seggern 2009; Curtis et al. 2014; Burke 2005; Burke/Greene/ McKenna 2016; Chawla 2016). Für unsere Erhebungen nutzen wir in Anlehnung an Imbke Behnken und J ürgen Zinnecker eine Methodenkombination aus mental mapping und einem zweistufigen Interviewverfahren (Behnken/ Zinnecker 2010), sogenannte narrative Landkarten. Dieses Vorgehen eignet sich nicht nur, um aktuelle Raumnutzungen unterschiedlicher Zielgruppen (hier Kinder und Familien) im Nahraum zu erforschen, sondern in unserem Fall auch, um retroperspektiv Tagesabläufe in den Anfangszeiten der Pandemie zu rekonstruieren: „Die narrativen Elemente sind grundsätzlich dazu geeignet, diezeitlichen, lebensgeschichtlichen Aspektestärker hervortreten zu lassen, die in der,Verräumlichung ' des Zeichenaktes eher zum Verschwinden gebracht beziehungsweise synoptisch auf einem Blatt zusammengeführt werden." (ebd.: 3) Da wir Alltagserfahrungen erfragten, die erst wenige Monate zurücklagen, ist davon auszugehen, dass den Befragten das gelebte Freiraumverhalten während der Frühphase der Coronapandemie noch vergleichsweise präsent war.

Befragt wurden insgesamt sechs Kind-Elternteil-Paare in zwei kleineren westdeutschen Großstädten zu ihrer Freiraumnutzung während der coronabedingten Schließungszeit zwischen Märzund Mai 2020. Diebeschriebenen Schließungen und Sperrungen brachten eine Veränderung von Routinen und besuchten Orten mit sich. In beiden Städten waren Schulen, Kindergärten und Kitas von Mitte Märzbis AnfangJ uni komplett geschlossen und wurden dann sukzessive wieder geöffnet (zunächst nur für Kinder „systemrelevanter“ Eltern). Die Spielplätze öffneten Anfang Mai. Neben den Spielplätzen waren 
Abb. 1 Karte K1 wohnungsnaher öffentlicher Freiraum Zuhause Mama

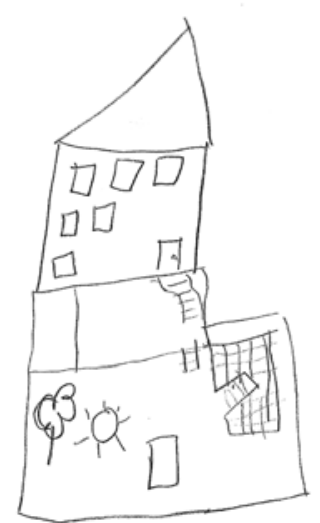

Abb. 2 Karte K4 Zeichnungen Wohnumfeld

Abb. 3 Karte K4 Zeichnungen Wohnumfeld mit Ergänzungen von Mutter und Interviewerin

(Quelle: Darstellungen Interviewmaterial)

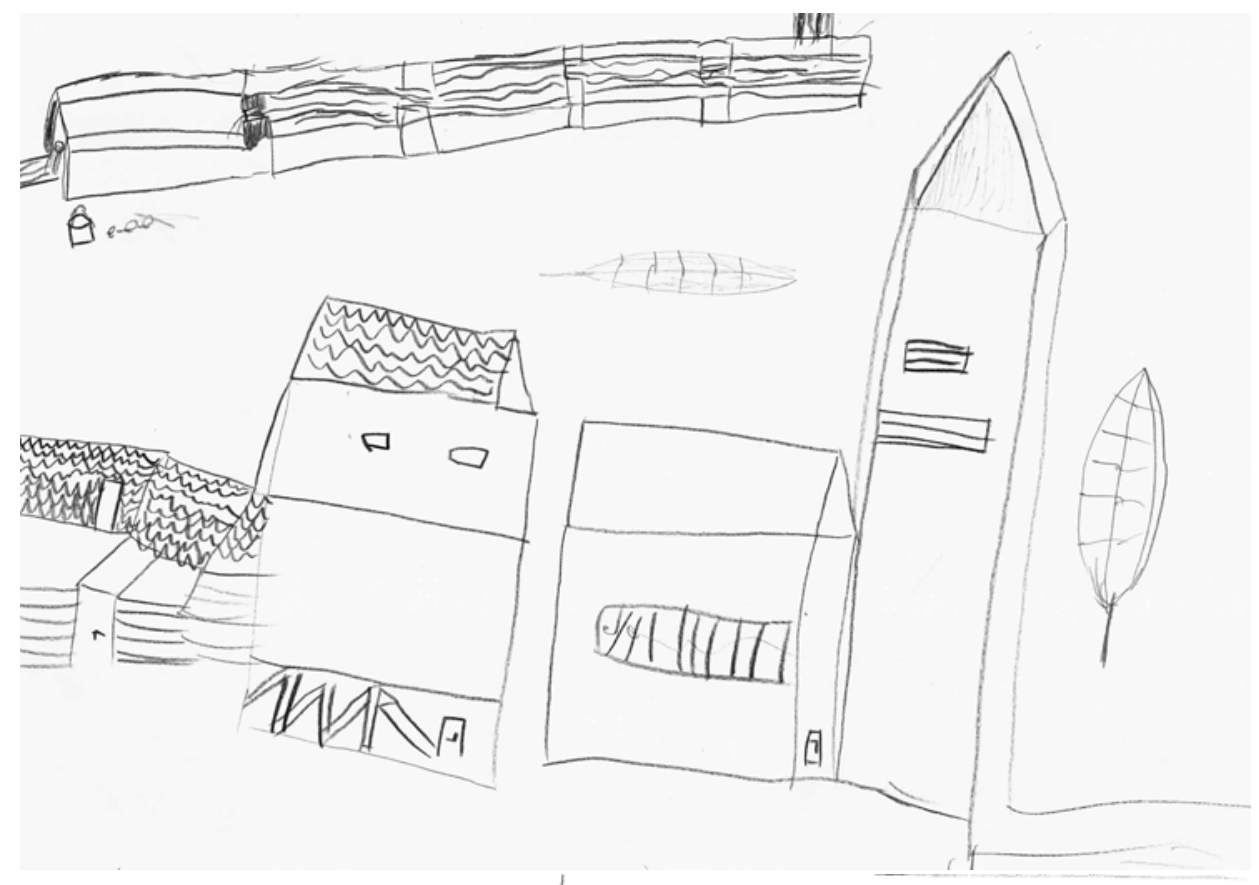

Tanenwäldchen
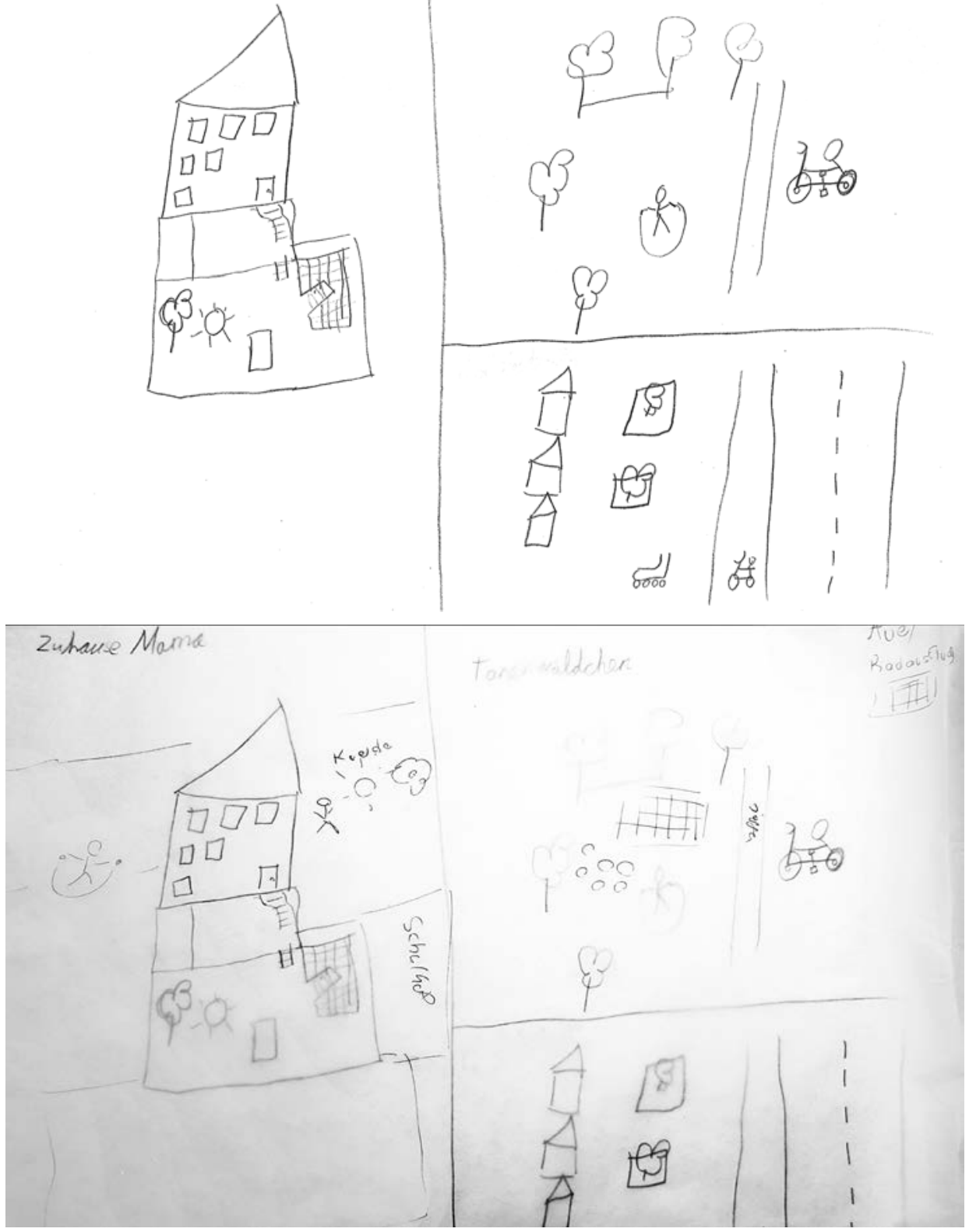
sogar ganze Parkanlagen zeitweise nicht zugänglich. Diese wurden erst wieder Anfang Mai für den Publikumsverkehr freigegeben.

Die befragten Kinder waren zwischen acht und zwölfJ ahre alt. Zwei Kinder sind Einzelkinder, drei haben jüngere Geschwister, ein Kind hatjüngere und ältere Geschwister. Die Befragungen fanden im Oktober 2020 bei den Familien zu Hause statt, in einem Fall im Hof des Wohngebäudes, was einevergleichbare, räumlich-konzentrierte Situation schuf. Nach einer Erläuterung des Vorgehens wurden zunächst die Kinder gebeten, ihren räumlichen Alltag während der Corona-Frühphase auf einem weißen A3-Blatt in Form einer Skizze zeichnerisch darzustellen und begleitend darüber zu berichten (vgl. Abb. 1-3). In einem zweiten Schritt wurde die Skizze besprochen und das Kind bekam dieMöglichkeit, dieZeichnung zu ergänzen. Anschließend wurde die Zeichnung des Kindes mithilfe eines separaten Transparentpapiers durch das anwesende Elternteil und die Interviewerin ergänzt (vgl. Abb. 2-3). Die Eltern waren in manchen Interviews still beobachtend, teilweise hielten sie sich im Nebenzimmer auf oder beschäftigten sich mit einem Geschwisterkind. Erst im Anschluss an die Befragung des Kindes wurden sie mithilfe eines leitfadengestützten Interviews ergänzend einbezogen.

Gegen ein gemeinsames Interview mit Kind und Elternteil spricht das Argument, dass Machtasymmetrien die Befragung beeinflussen können - zum Beispiel, wenn Kinder in Anwesenheit der Eltern nicht offenbaren, dass sie mehr Zeit mit Medienkonsum verbracht haben, als in der Familie vereinbart war. Diese Gefahr erschien uns jedoch bei den relativ jungen Kindern nicht sehr gravierend, da diese - gerade während der Ausgangsbeschränkungen ohnehin viel unter der Aufsicht von Erwachsenen standen und Medienkonsum kaum selbstständig stattfand. Eine Ausnahme stellt das Gespräch mit einer Zwölfjährigen dar, die sehr freimütig über ihre Aktivitäten mit dem Smartphone sprach und deren Angaben vom anwesenden Elternteil bestätigt wurden. Aus unserer Sicht überwogen die Vorteile dieses Settings: Erstens gab dieAnwesenheit eines Elternteils den noch relativ jungen Kindern, diedie Interviewerin nicht oder nur sehr flüchtig kannten, ein Gefühl der Sicherheit (vgl. Million 2021a). Zweitens konnten sich Kinder und Eltern im Gespräch gemeinsam erinnern und ihre Aussagen jeweils ergänzen. Gleichwohl ist es gut möglich, dass subjektiv konfliktvolle Momente nicht vor den Eltern (aus Kindersicht) beziehungsweisevor den Kindern (aus Elternsicht) thematisiert wurden. Da innerfamiliäre Konflikte aber nicht den Kern unserer Forschungsfragen betreffen, haben wir in Kauf genommen, hier möglicherweise weniger zu erfahren und dafür durch die gemeinsame Befragung einen besseren Überblick über die Aktivitäten und genutzten Orte zu erhalten.

Alle Interviews wurden in der Auswertung anonymisiert. Für die Interpretation wurde eine synthetisierende Auswertungsmethode gewählt, mit der die räumliche und die prozessuale Seite der Untersuchung zusammengeführt wurden (Behnken/Zinnecker 2010: 20 f.; Million 2021a). Ergänzt wurden diese Daten durch Informationen zur Nachbarschaft und zur Wohnsituation der Kinder (Fotos, Plan der Nachbarschaft, Information zu Größe, Lage und Grundriss der Wohnung). Die Zeichnungen der Kinder, die ergänzenden Transparentpapierzeichnungen, die Transkriptionen der Audioaufzeichnungen der Interviews sowie die Reflexion des Zeichenund Interviewprozesses wurden in einem wissenschaftlichen Quellentext 
aufbereitet. Zudem wurden die Angaben der Befragten durch Vergleich, Übertragung, Übersetzung und Überlagerung in die Stadtpläne integriert (vgl. Million 2021a). Diese verschriftlichten Zwischenprodukte bilden die Grundlage für die im Folgenden präsentierten Ergebnisse. Im Verlauf der Untersuchung zeigten sich einige methodische Probleme und Besonderheiten, die in der abschließenden Reflexion der Ergebnisse thematisiert werden.

\section{Basteln, matschen und toben im wohnungsnahen Freiraum: Ergebnisse}

Die referierten Studien haben gezeigt, dass Familien in der Pandemie-Frühphase auf viele sonst alltägliche Räume und wichtige Kontakte verzichten mussten. Die Themen Verlust und Verzicht lassen sich auch in unserem empirischen Material ablesen. Auf die Frage, welche Orte und Aktivitäten sie vermisst haben, nannten fast alle Kinder als Erstes Schule und Hort. Zudem war für sie der Ausfall von Vereinssport und die Schließung der Spielplätze belastend und sie vermissten Ausflüge ins Schwimmbad, in Kino oder in die Kletterhalle beziehungsweise Indoor-Spielhalle. Vor allem der fehlende Kontakt zu Gleichaltrigen stellte für sie eine Beeinträchtigung dar. Entsprechend wurde in allen Gesprächen mit Familien mit mehreren Kindern thematisiert, wie positiv es gewesen sei, in dieser Situation Geschwister gehabt zu haben. Auch die Großeltern, die während dieser Phase von keiner Familie besucht wurden, wurden von den Kindern vermisst. Ebenso fielen kleinere selbstständige Besorgungen weg, wieder Gang zum Bäcker oder zum nächsten Supermarkt, um mit befreundeten Kindern Süßigkeiten zu kaufen. Bei zwei Familien konnte ein geplanter Urlaub nicht stattfinden.

Übereinstimmend berichteten die Befragten, dass ihr Aktionsradius während der Pandemie-Frühphase deutlich kleiner geworden sei. Das betraf dieKinder nochstärkerals dieErwachsenen, diewenigstens Einkäufemachen oder sich an den Arbeitsplatz begeben „durften“: „[...] weil wir sonst ja immer auch viel unterwegs sind und Verwandte, Freunde besuchen innerhalb der Stadt. Und auch sonst viel unterwegs sind und Freunde besuchen in anderen Städten. Undjetzt waren wir wirklich nur hier."(E5) DieKinder wurden auch nicht mehr zu alltäglichen Besorgungen mitgenommen: „Wir durften nicht mal mit zum Einkaufen, das war richtig fies!“ (K6)

Öffentliche Verkehrsmittel wurden kaum bis gar nicht mehr genutzt, dafür wurden kürzere Strecken häufiger mit dem Fahrrad zurückgelegt. Bei längeren Strecken nutzten die meisten Familien das Auto, insbesondere wenn die Kinder dabei waren. Insgesamt waren dieKinder aber weniger mit dem Auto unterwegs als sonst.

Neben den beschriebenen Verlusten werden aber auch viele Anpassungen in den Alltagsabläufen und der Freiraumnutzung deutlich. Beim Blick auf die Zeichnungen und Stadtplänemit den übertragenen Angaben der Kinder fällt auf, dass sowohl der wohnungsnahe und der von der Wohnung unabhängige öffentliche Freiraum als auch die Innenräume intensiver genutzt wurden als sonst. Im Folgenden ordnen wir die Aussagen der Interviews nach den Räumen, in denen sich die Kinder vorrangig aufgehalten haben und beschreiben die Tätigkeiten, die sie dort ausgeführt haben. Dafür orientieren wir uns 


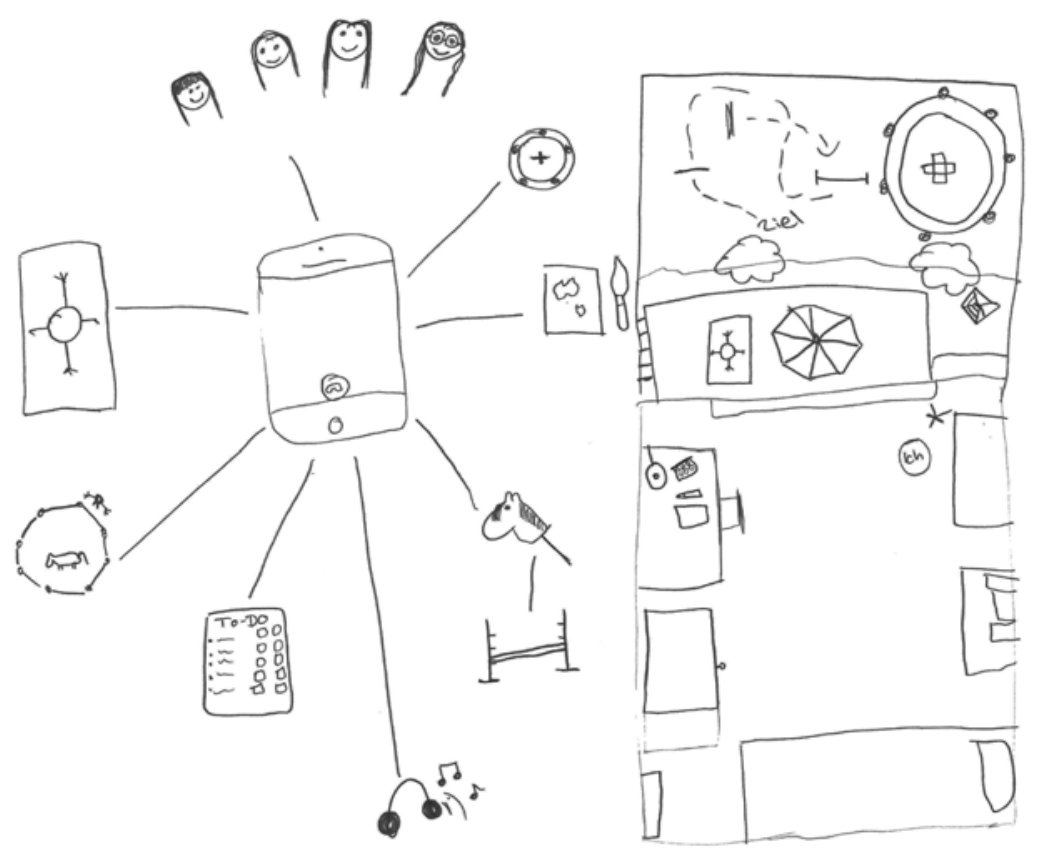

Abb. 4 Karte K3 Smartphone und Kinderzimmer (Quelle: Darstellung Interviewmaterial)

an der in der Freiraumplanung üblichen Zonierung nach der Zugänglichkeit der Räume (Schmitt/Sommer/Wiechert 2014: 19 ff.). Wir wählen dabei die Raumkategorien „Innenräume“, „Übergangsräume“, „privat, gemeinschaftlich oder öffentlich nutzbare Freiräume in Wohnungsnähe“ und „von der Wohnung unabhängige Freiräume“. Mithilfe von Interviewauszügen zeigen wir verschiedene Perspektiven auf die jeweiligen Raumkategorien auf.

\section{Innenräume}

Bis auf ein Kind hatten allebefragten Kinder ein eigenes Zimmer. Das älteste befragte Kind (zwölf J ahre) hielt sich vornehmlich in seinem Zimmer auf und beschäftigte sich mit dem Smartphone, es telefonierte und chattete mit Freund_innen, machte Schularbeiten, schaute Bastelvideos und hörte Hörbücher. Dies spiegelt sich auch in seiner Zeichnung wider, in der die Wichtigkeit des Smartphones durch die zentrale Position und seine relative Größe zum Ausdruck kommt (vgl. Abb. 4). Diesem Kind waren die Privatsphäre und die Möglichkeit zum Rückzug sehr wichtig. Jüngere Kinder thematisieren dies nicht so sehr, sie nutzten auch den Rest der Wohnung für ihre Aktivitäten.

„Und dann sind wir da immer drauf und dann immer so hin und her gewippt und wir durften nicht so runterfallen. Manchmal haben wir uns auch so einen Zug gebaut aus Stühlen und haben dann damit gespielt und dann haben wir so ein Spielpferdchen damit transportiert und haben mit Duplo so eine Stadt oder ein Dorf gebaut. "(K1)

„[...] aber wir spielen auch mit [meiner kleinen Schwester] hier im Wohnzimmer oder lesen ihr was vor, da auf dem Sofa oder so“ (K4).

Das Wohnzimmer wurde zudem für Aktivitäten genutzt, die unter der Aufsicht der Eltern stattfanden (fernsehen/am Tablet spielen) oder an denen sich die ganze Familie beteiligte (Gesellschaftsspiele). In einer Familie hatte der lange Flur in der Wohnung eine große Bedeutung, da die Kinder 
dort Rollenspiele spielten. Langeweile stand weniger im Vordergrund als erwartet und wurde meist in Verbindung mit dem Vermissen der Freund_ innen angesprochen:

„Und wollte einfach nur wieder Freunde sehen, aber ging ja nicht. Und dann waren wir halt zu Hause und [haben] halt auch ziemlich [viel] einfach nur drin rumgesessen. Wusste nicht, was ich [...] tun soll. [Dann habeich] abgewartet, bis mir irgendwie wieder was eingefallen ist. Und dann hat man sich halt irgendwas ausgedacht." (K5)

\section{Übergangsräume}

In mehreren Familien wurden Übergangsräume wie Treppenhäuser, Hausflure, Balkone oder Fenster thematisiert. Sie waren relevant als verbindendes Element zwischen Innen- und Außenraum und wurden zur Erschließung und teilweise auch als Aufenthaltsortegenutzt. Mithilfeakustischer oder visueller Signale konnten durch diese Übergangsräume einerseits die Eltern die Aufsicht über die Kinder behalten, auch wenn sie sich selbst in der Wohnung aufhielten und parallel anderen Aufgaben nachgingen. Andererseits konnten die Kinder hier mit anderen Kindern Kontakt aufnehmen, zum Beispiel um zu kommunizieren, dass gemeinsames Spielen möglich ist:

„Wenn das Tor offen ist und wenn es zu ist. Also wenn das Tor offen ist, dann hat [mein Freund] eigentlich Zeit. Und wenn es zu ist, dann hat er eigentlich keine Zeit." (K2)

Der Balkon wurde oftmals zum Austausch mit anderen Kindern genutzt, zum Kontakthalten und als Abkürzung nach draußen (vgl. Abb. 5):

„Wir haben am Balkon so eine Schnur und da haben wir, also ich und [meine Freundin], die ist oben in der Wohnung, wir sind Freundinnen und wir haben da halt also so Briefe geschrieben und sie dann halt an der Klammer an die Schnur gemacht und dann konnte derjenige das so hochziehen und runter.“(K6)

Abb. 5 Karte K2 Strickleiter vom Balkon in den Garten (Quelle: Darstellung Interviewmaterial)

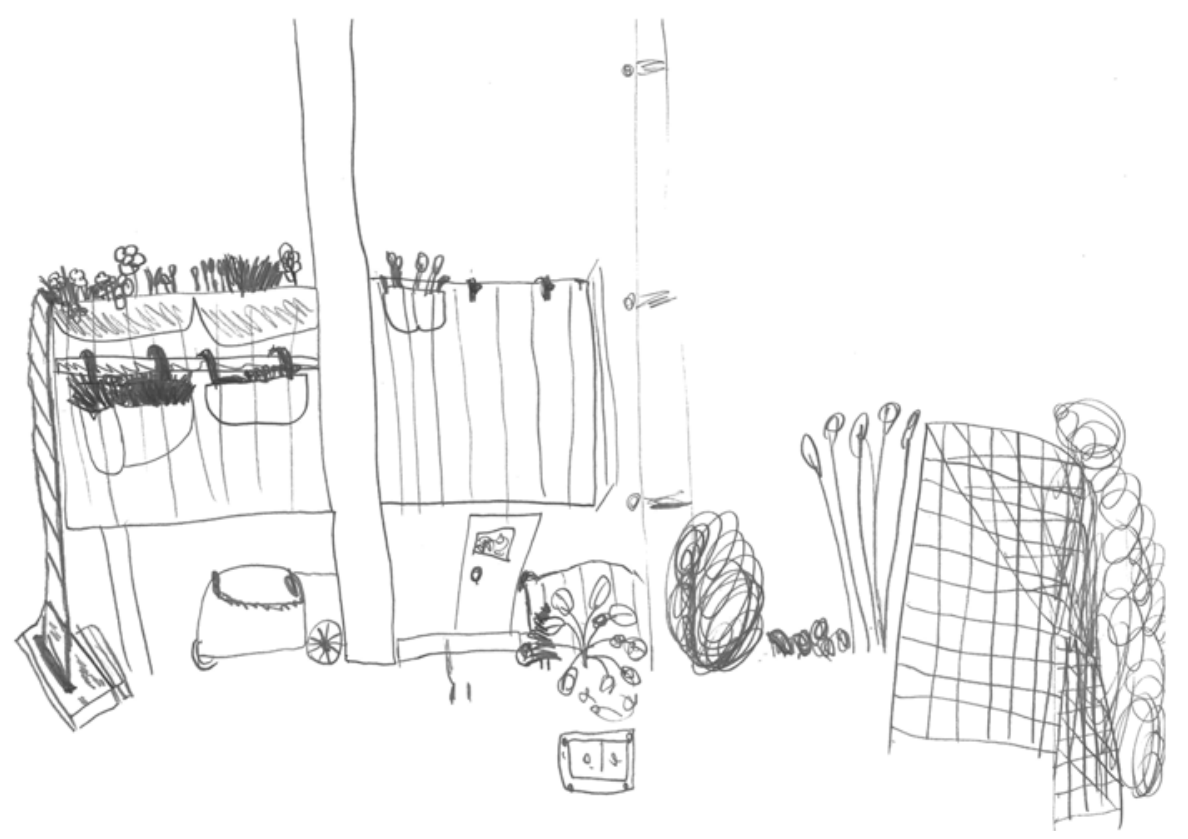


Privat, gemeinschaftlich oder öffentlich nutzbare Freiräume in Wohnungsnähe

Erwartungsgemäßhielten sich dieKinderinsbesondere in privaten oder von mehreren Parteien genutzten Gärten und Höfen auf, sie spielten aber auch auf Gehwegen, nahe gelegenen innerstädtischen Plätzen und in einem Fall auf einem an den Hinterhof angrenzenden (eigentlich abgesperrten) Schulhof. Nur eins der befragten Kinder wohnt in einem frei stehenden Einfamilienhaus mit eigenem Garten, eins in einem Reihenhaus. Die anderen Familien leben in Mehrparteienhäusern, wo sie einen Hinterhof oder kleinere, gemeinschaftlich genutzte Gärten zur Verfügung haben. Die Kinder ohne eigenen privaten Garten nutzten insgesamt mehr Orte und verließen häufiger das Grundstück zum Spielen (vgl. Abb. 6).

Die Aktivitäten im wohnungsnahen privaten Freiraum waren vielfältig und umfassten eine große Bandbreite an Sportarten und Bewegungsspielen (Ballspiele wie Fußball, Federball und Basketball, Trampolinspringen, Seilspringen, Hobby Horsing, Bogenschießen, Yoga, Fahrrad und Inliner fahren, Joggen), die Beschäftigung mit Tieren (Kaninchen, die „Nachbarschaftskatze“,

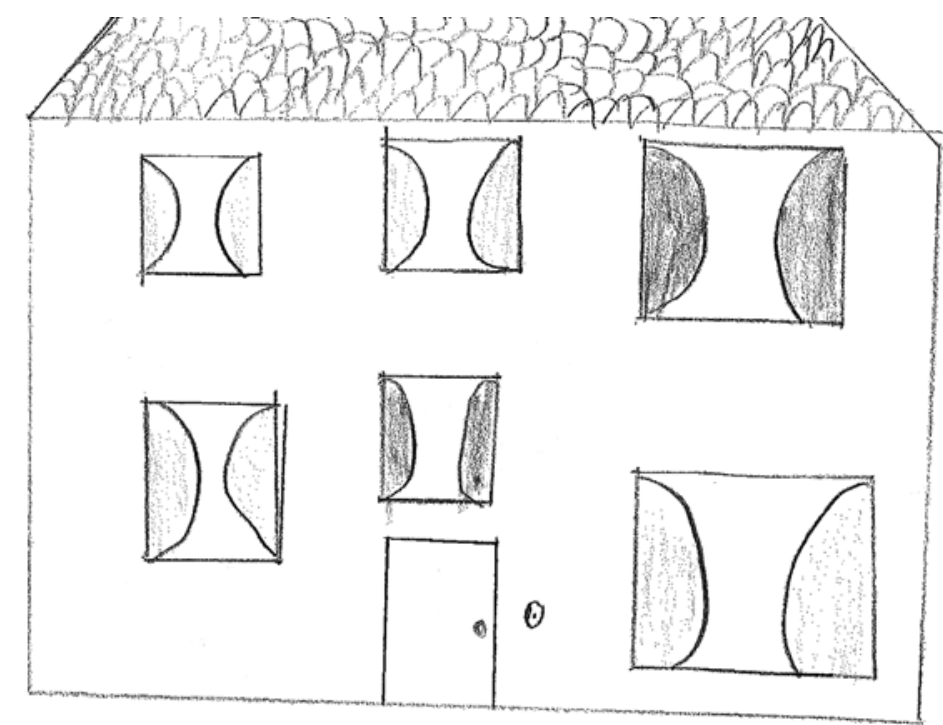

Abb. 6 Karte $\mathrm{K} 6$ Haus und Garten als wichtigster Spielort (Quelle: Darstellung Interviewmaterial)
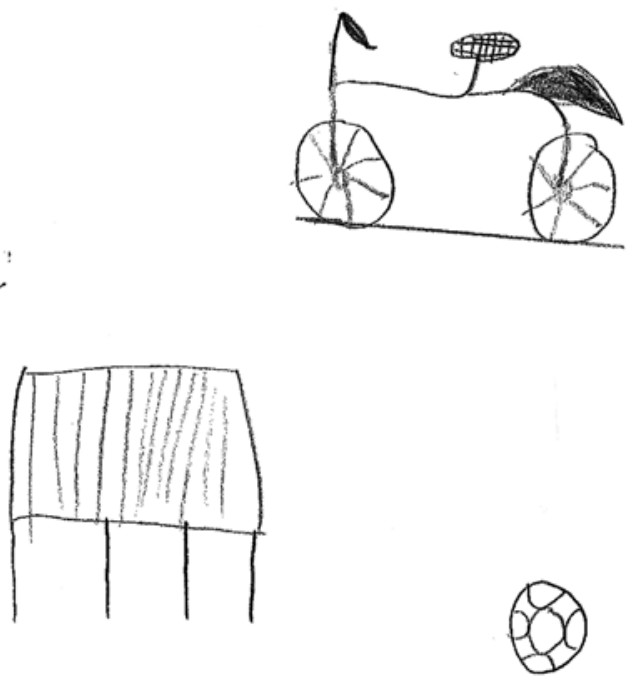
Abb. 7 Karte K2 Spiellandschaft im Gemeinschaftshof (Quelle: Darstellung Interviewmaterial)

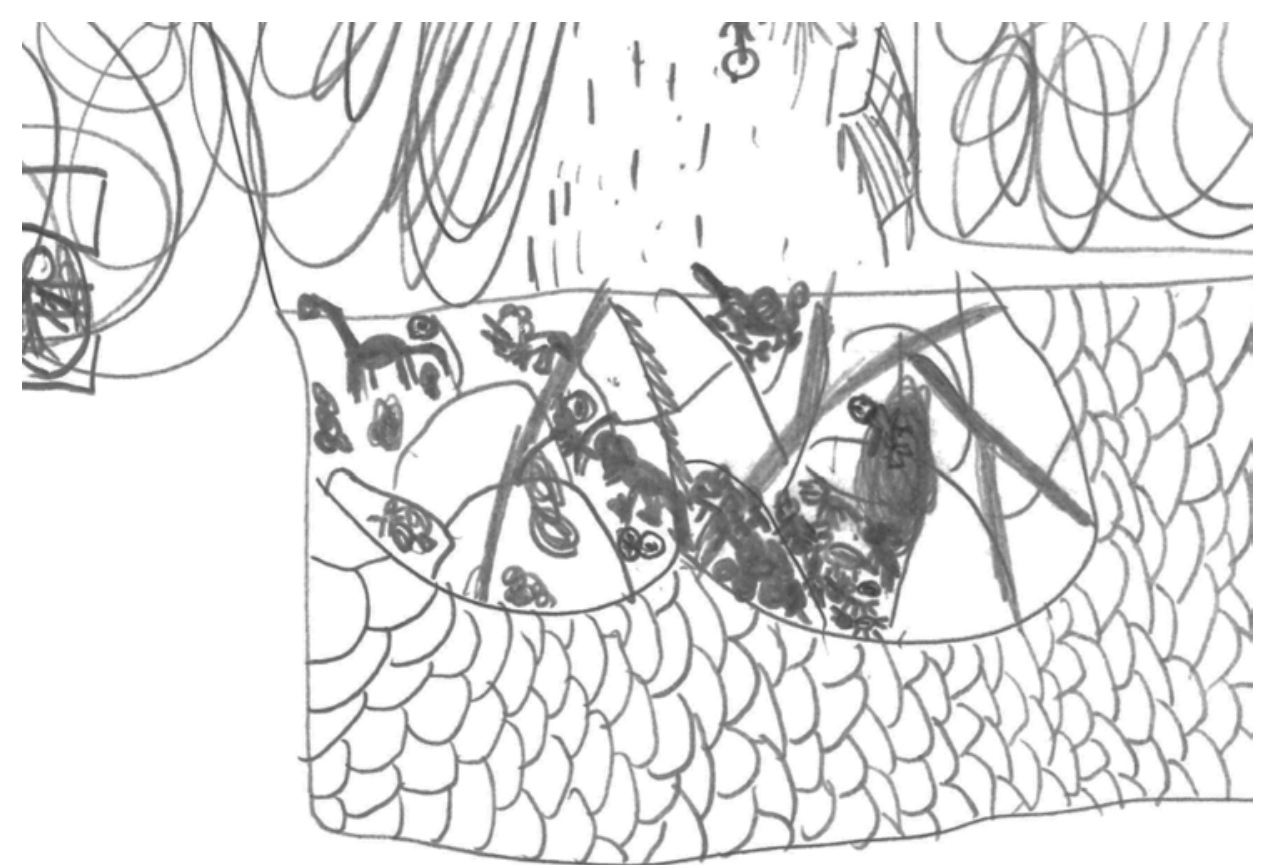

verschiedene Insekten), Rollenspiele (Bauernhof, Reiterhof, Verkleiden mit Kartons) oder Gartenarbeit zusammen mit den Eltern. Daneben nahm das Spielen mit Sand, Matsch und/oder Wasser, das Anlegen von Landschaften mit den vorgefundenen Elementen der genutzten Orte wie Gullideckel, Pfützen, Steine oder Bewuchs sowie das Malen mit Kreide einen großen Stellenwert ein (vgl. Abb. 7). Teilweise vertieften sich die Kinder mehrere Tage lang in solche Spiele:

„Ich weiß, mit was wir zuerst draußen gespielt haben. Mit PlaymobilZwergen und Trollen. [...] Damit haben wir hier auf dem Rasen gespielt und dann auch in der Burg aus Stein, die hier irgendwo war. [...] Und hier war dann irgendwo das Verlies. Da haben wir [...] für die Dinos was gebaut und halt mit Kreide auch was aufgemalt. Ich glaube, hier war Wasser. Hier war ein Fluss. Das war noch ein See. Und dann hier [...] mit Steinen." (K2)

Die Kinder bespielten außerdem gemeinschaftlich oder öffentlich nutzbare wohnungsnahe Freiräume wie Höfe, Gemeinschaftsgärten, Abstandsgrün oder Gehwege. Viele der im wohnungsnahen privaten Freiraum ausgeführten Tätigkeiten wurden, wenn dieser Raum zu klein oder nicht vorhanden war, in den gemeinschaftlich genutzten oder öffentlichen Freiraum verlegt. Diese Freiräume kompensierten somit teilweise den fehlenden privaten Freiraum und wurden stärker frequentiert als vor der Pandemie. Insgesamt scheint die Wertschätzung für den wohnungsnahen Freiraum gestiegen zu sein, die Nutzung wurde intensiviert, bewusster gestaltet und genossen.

„Wir schimpfen immer über unseren kleinen, dunklen Hinterhof, aber während der Coronazeit war er wirklich Gold wert.“(E6)

„Also hier draußen bei uns im Garten, [...] da spielen die aber sowieso immer schon auch und haben das einfach viel mehr dann genutzt, hier Rad zu fahren, Inliner zu fahren, mit Kreide herumzumalen und so, also die Sachen, dieschon da waren, einfach dann mehr genutzt."(E1) 


\section{Von der Wohnung unabhängige Freiräume}

Nicht nur wohnungsnahe, sondern auch von der Wohnung unabhängige Freiräume erhielten für die befragten Familien während der Schließzeiten eine größere Bedeutung.

„Wir haben sehr zu schätzen gelernt, wie grün wir hier eigentlich wohnen, also wir waren so viel in [den großen Stadtparks] und überall unterwegs wie eigentlich noch nie zuvor.“(E6)

Auch neue Orte und Aktivitäten wurden entdeckt, so zum Beispiel ein kleinerer Nachbarschaftspark, den die Familie vorher nicht aufgesucht hatte, oder sogar Parkplätze, die während der Pandemie weniger stark genutzt wurden und daher Platz für die Aktivitäten der Kinder boten.

Die verschiedenen Zonen des Wohnumfelds wurden von den Kindern für unterschiedliche Betätigungen genutzt und angeeignet, wobei der Innenraum vornehmlich für Schulaufgaben und ruhige Aktivitäten verwendet wurde, während Bewegungsspiele oft in den wohnungsnahen Freiraum verlegt wurden. Hier spielen die Verknüpfung von Innen- und Außenraum sowie die Ausprägung der Übergangsräume vor allem für die Aufsicht der Eltern eine Rolle. Die Möglichkeit, Ausflüge in den von der Wohnung unabhängigen Freiraum zu unternehmen, hing von den zeitlichen Ressourcen der Eltern ab, der erweiterte Aktionsradius um die Wohnung herum zudem von Alter und (zugetrauter) Selbstständigkeit der Kinder.

Unsere Ergebnisse zeigen deutlich, dass neben dem gefühlten und tatsächlichen Verlust des Zugangs zu Freiräumen bei Kindern und Familien eine Vielzahl unterschiedlicher Anpassungsprozesse stattfand. Diese Prozesse sowie die damit verbundenen Kompensationsstrategien diskutieren wir im folgenden Abschnitt.

\section{Räumliche und zeitliche Kompensationsstrategien im Familienalltag: Diskussion}

Über eine Analyse der Ergebnisse haben wir verschiedene Strategien zur Kompensation der unfreiwilligen pandemiebedingten Einschränkungen identifiziert, die wir im Folgenden näher beleuchten. Wir haben sie in fünf Kategorien erfasst, wobei manche Strategien zu mehreren dieser Kategorien passen: Routinen, Ausflüge, Anschaffungen, Nutzung digitaler Räumeund das Ermöglichen analoger Kontakte trotz Kontaktbeschränkungen.

\section{Routinen}

Die Mehrheit der befragten Familien versuchte eine gewisse Normalität und gewohnte Routinen aufrechtzuerhalten. Dabei wurde die Verfügung über die eigeneZeit als positiv wahrgenommen, was sich auch in den oben genannten Studien nachvollziehen lässt (Andresen et al. 2020a). So konnten dieKinder zwar etwas länger schlafen, wurden danach aber zunächst dazu angehalten, die Hausaufgaben zu erledigen. Danach konnten sie wieder spielen. 
„Und da haben wir auch probiert, irgendwie den Rhythmus aufrechtzuerhalten, morgens ganz normal aufstehen und dann [...] spätestens halb neun saß [das Kind] auch mit seinen Aufgaben erst mal da. Und das wurde erst mal vormittags sozusagen durchgezogen, bis er dann spielen durfte." (E2)

In einigen Familien entstanden neue Routinen, in die der wohnungsnahe Freiraum integriert wurde, zum Beispiel das morgendliche Füttern der „Nachbarschaftskatze" oder das Trampolinspringen in den Lernpausen. In einem Fall leben die Eltern getrennt, die Kinder teilen sich die Zeit zwischen beiden Elternteilen auf, sind aber häufiger bei der Mutter. Während der Schließzeit mussten die Mutter und ihr Partner zumindest zeitweise am Arbeitsplatz anwesend sein, wohingegen der Vater von zu Hause arbeiten konnte. Daher wurde die Aufenthaltsregelung flexibler gestaltet als sonst und die Kinder waren öfter beim Vater. Einzig der Tagesablauf des zwölfjährigen Kindes änderte sich komplett: Es schlief bis zum späten Vormittag, beschäftigte sich hauptsächlich digital und verließ sein Zimmer kaum noch.

Ein weiterer Effekt war eine gefühlte Entschleunigung des Alltags, die Möglichkeiten für mehr gemeinsam verbrachte Familienzeit und längere Aufenthalte im Freien eröffnete.

„Dadurch, dass man sich nicht mit anderen Leuten verabredet hat und keine Termine im Kalender durch die ganzen Freizeitaktivitäten, war ja der Nachmittag auch frei. Und dann haben wir halt immer so was zusammen gemacht. Und das ist eigentlich so was, das hatte so eine Regelmäßigkeit und das haben wir leider nicht darüber hinaus gerettet, aber das war schön." (E5)

„Aber irgendwie ging das trotzdem ganz gut, also wir haben dann einfach auch andere Sachen gemacht, also wir waren dann viel draußen, viel [...] spazieren." (E1)

„[Unsere Draußen-Spielsachen haben wir vorher] halt nicht so genutzt eigentlich. Weil wir halt nicht so oft sonst draußen waren, weil [meine ältere Schwester] musste ja auch, sie ist jetzt auch schon im Gymnasium, und damusstesieauchimmerviel Hausaufgaben machen und da waren wir halt nicht ganz so viel draußen. "(K4)

Nach Aussagen der Eltern haben einige Kinder während der Schulschließung gelernt, sich besser alleine zu beschäftigen und ihre Aufgaben für die Schule eigenständiger zu organisieren. Sie haben sich stärker ins Spiel vertieft und auch gelernt, sich draußen besser selbstständig zu bewegen, zum Beispiel durch häufigeres Fahrradfahren. Insgesamt haben die Schulpause und der weniger durchgetaktete Alltag auch Entwicklungspotenziale freigesetzt:

„Also[das Kind] war auch entstresst dadurch, dass es diesen Schulstress nicht mehr hatte, das hat ihm [...] ganz gutgetan und es hat, nachdem es dann wieder an der Schule war, auch die Rückmeldung bekommen, dass es einen total dollen Entwicklungsschub hatte.“(E1) 
Fast alle befragten Eltern (eine Ausnahme) organisierten die Kinderbetreuung zusätzlich zu ihrer Erwerbstätigkeit und ohne Hilfe von außen:

„Also wir hatten das aufgeteilt, da wir gemerkt haben, dass für das Homeschooling eigentlich immer einer dabei sein musste, der das einteilt mit den Aufgaben, dann hat entweder [der Vater] oder ich den Dienst gemacht. Mit dem Homeschooling, wirhaben dann unsere Arbeitszeit drum herum geplant. Deswegen war dann der ganze Tag voll. Aber es hat dann immer einer Zeit gehabt, das zu machen. " (E5)

Die Stimmung in der Familie war dadurch zunehmend angespannt, die Familienmitglieder wurden als „dünnhäutig“ bezeichnet, der Spagat zwischen Arbeit und Kinderbetreuung wurde, wie sich auch schon in den oben genannten Studien abzeichnet, als „wirklich schwer“ empfunden (E1). Immerhin konnten die meisten Eltern von zu Hause arbeiten und sich die Arbeit zeitlich relativ flexibel organisieren.

\section{Ausflüge}

Eine weitere Kompensationsstrategie waren Ausflüge. So gab es zusätzlich zum wohnungsnahen Freiraum eine Reihe von öffentlichen Orten, die weiter entfernt liegen und von den Familien bewusst als Teil der Freizeitgestaltung aufgesucht wurden. In einer der Städte nannten alle Befragten die zwei großen Stadtparks, außerdem wurden Ausflüge an einen Fluss gemacht, zum Beispiel zum Inlineskaten oder Spazierengehen. Einige Familien nutzten Wanderwege außerhalb der Stadt oder machten Fahrradtouren. Dass diese Strategie insgesamt viele Menschen verfolgten, verdeutlicht der folgende Gesprächsausschnitt:

„Ich dachte, da treffen wir nicht so viele, aber da war dann auch ziemlich viel los, ehrlich gesagt, [...] plötzlich Highway [am Fluss] entlang.“ (E2)

In der anderen Stadt wurden ebenfalls Orte genannt, die pandemiebedingt auffällig hoch frequentiert waren, so zum Beispiel der Stadtwald und ein See. Zwei der befragten Familien erzählten aber auch von Spaziergängen außerhalb der Stadtgrenzen sowie an nahe gelegenen Feldern, die sie unter anderem deshalb als Ziel auswählten, um den viel besuchten Ausflugsorten auszuweichen und sich so besser an die Abstandsregeln halten zu können.

\section{Anschaffungen}

$\mathrm{Zu}$ den Anschaffungen, die bewusst wegen der Kontaktbeschränkungen gemacht wurden oder zufällig zeitlich damit zusammenfielen, gehören Trampoline, Fahrräder und Spielsachen für draußen. Eine Familie kaufte Kaninchen für die Kinder, eineandere bastelte gemeinsam Steckenpferdeund Zubehör dafür.

„Dakonnteich dann auch [...] mit spielen, also [das Kaninchen] füttern und, weil ich ja nicht rauskonnte, das war dann schon so was, wo ich mich mit ablenken konnte von Corona.“ (K6) 
„Die Kinder haben im Lockdown auch noch mal neue Fahrräder bekommen, weil die[alten] zu klein waren. [...] Da mussten wir drei Stunden warten, bis wir überhaupt in den Laden erst mal reingekommen sind." $(\mathrm{E} 4, \mathrm{~K} 4)$

„[Mit dem Trampolin] haben wir uns eine Kinderbetreuung in den Garten gestellt." (E5)

\section{Nutzung digitaler Räume}

Zudem durften die Kinder verstärkt digitale Räume nutzen, zum Beispiel länger fernsehen oder am Computer oder Tablet spielen. Auch Fitnessübungen wurden in der Wohnung gemacht, meist mit Anleitungsvideos. Über Telefon und Videochat wurde Kontakt zu Freund_innen und Großeltern gehalten:

„Ich habe das Telefon dann auch mal irgendwo drangestellt, dass wir uns auch sehen, und dann haben wir was gemeinsam gebastelt.“ $\left(\mathrm{K}_{3}\right)$

Vor allem das älteste befragte Kind verbrachte viel Zeit mit dem Smartphone und organisierte darüber seinen digitalen Austausch mit Freund_innen. Sie veranstalteten Bastel-, Bewegungs- und Rollenspiele, wobei die Kompetenz, die virtuellen und materiellen Ebenen miteinander verschmelzen zu lassen, auffallend hoch war. Gemeinsam mit den Freund_innen wurden Landschaften mit Spielfiguren auf dem Fußboden aufgebaut, sich gegenseitig per Videochat gezeigt und in dieser Form auch gemeinsam bespielt - selbst Yoga und Trampolinspringen wurden gemeinsam per Videochat praktiziert (vgl. Abb. 4).

\section{Ermöglichen analoger Kontakte trotz Kontaktbeschränkung}

Der analoge Kontakt mit anderen Kindern war bei den befragten Familien sehr unterschiedlich ausgeprägt: Mehrere Kinder und Eltern berichteten, sich mit einer anderen Familie, meist aus der Nachbarschaft, zusammengetan zu haben, um die Kinderbetreuung gemeinsam zu bewältigen und den Kindern das Spielen mit anderen Kindern zu ermöglichen. Ein Kind wurde jeweils mehrere Tage pro Woche tagsüber bei einer anderen Familie betreut, da die Eltern nicht von zu Hause arbeiten konnten. Andere hielten sich wiederum sehr strikt an dieKontaktbeschränkungen und sprachen sich sogar innerhalb des Hauses mit den anderen Familien ab, wer zu welchem Zeitpunkt die Gemeinschaftsflächen nutzen würde, damit man sich nicht begegnete.

Neben den virtuellen Kontaktmöglichkeiten wurden auch analoge Strategien ohne direkten Körperkontakt genutzt - wie die erwähnte Balkonpost. Darüber hinaus wurdedas Spiel mit den wenigen erlaubten Kontaktpersonen wie Geschwistern oder Nachbarskindern intensiviert.

„Also es gab natürlich auch Zank in der Zeit, aber unterm Strich habe ich [...] das Fazit gezogen, dass die beiden [Geschwister] zusammen auch gut funktioniert haben und auch so eine gewisse Ruhe nach einer Zeit war."(E6) 
„Was ich beobachten konnte, war, dass die beiden [Nachbarskinder] da sich so ein bisschen zusammengerauft haben [...], es war schon fast so geschwistermäßig. Also es gab ab und zu mal Zoff, aber das ging dann auch wieder. [...] Die haben gemerkt: ,Moment, das ist gerade so der Einzige, mit dem ich hier was machen kann', und haben sich dann irgendwie auch wieder arrangiert. Und das war so ein Selbstläufer. Also gerade wenn schönes Wetter war und die haben da draußen ihre Festung aufgebaut oder irgendwas, die waren komplett in einer anderen Welt.“(E2)

Die vorgestellten Kompensationsstrategien im Familienalltag verdeutlichen die hohe Flexibilität, gute Anpassungsgabe und den großen Erfindungsreichtum der Kinder und Eltern im pandemiebedingten „neuen Alltag“. Zudem hat sich gezeigt, dass der digitale Raum insbesondere bei relativ jungen Kindern nur bis zu einem gewissen Punkt „,analoge“ soziale Kontakte ersetzen kann. Zwar waren alle befragten Familien froh über die zusätzlichen digitalen Möglichkeiten des Kontakts, betonten aber gleichzeitig, wie sehr sie Freund_innen und die erweiterte Familie vermissten. Mit zunehmendem Alter schwächt sich dieser Effekt möglicherweise etwas ab, wie das Beispiel des zwölfjährigen Kindes nahelegt.

Der wohnungsnahe Freiraum spielte in allen Familien eine wichtige Rolle und schuf Entlastung im stressigen Alltag. Es ist davon auszugehen, dass insbesondere die aufwendigeren oder kostspieligeren Strategien wie Anschaffungen und Ausflüge insbesondere von ressourcenstarken Familien gewählt wurden, in denen die Eltern ihre Arbeitszeit flexibel gestalten konnten und keine finanziellen Probleme bestanden. In eher ressourcenarmen Familien, in denen zudem keine flexible Arbeitszeitgestaltung möglich war, bekam der öffentliche wohnungsnahe Freiraum und dessen Ausstattung somit eine noch größere Bedeutung innerhalb der Möglichkeiten, die den Familien zur Kompensation zur Verfügung standen.

Interessant ist, dass in allen Familien neben den genannten Schwierigkeiten auch positive Effekte der Situation benannt wurden. Einerseits wird die große Anstrengungsleistung deutlich, wenn Schule, Arbeit und Familienleben im beschränkten Raum der eigenen Wohnung und dem wohnungsnahen Freiraum stattfinden. Andererseits wird die neu gewonnene Flexibilität in der Tagesroutine und die intensiv zusammen verbrachte Zeit als positiv angesehen.

\section{Kartieren und Erinnern: Methodische Reflexion}

Ebenso wie der Familienalltag der befragten Kinder war auch die vorliegende Untersuchung selbst von der Sondersituation der Pandemie beeinflusst. Im Zuge der Durchführung und Auswertung der Interviews entstanden einige methodische Herausforderungen. So erschwerten zum Zeitpunkt des Feldzugangs und der Durchführung der Interviews die erhöhten Infektionszahlen der zweiten Welle die Forschung. Aufgrund von Vorsichtsmaßnahmen und erneut eingeführten Kontaktbeschränkungen standen angesprochene Familien den Interviewanfragen zögerlich bis ablehnend gegenüber, manch bereits organisiertes Interview wurde wieder abgesagt. In einigen Fällen hatten Kinder schlicht keine Lust, sich an der Studie zu 
beteiligen. Pandemiebedingt konnten die Kinder nicht direkt angesprochen werden, sodass wir auf die Eltern als Vermittlungspersonen angewiesen waren. Dies brachte natürlich eine verminderte Kontrolle über die Art und Weise der Anfrage mit sich. Was die Eltern den Kindern erzählten und wie sie die Möglichkeit zur Teilnahme einführten, entzieht sich unserer Kenntnis.

Um die Studie dennoch durchführen zu können, verlegten wir uns auf Kontakte aus unserem erweiterten privaten Netz, was die sozioökonomische Diversität der befragten Familien beeinflusste. Entgegen unserer ursprünglichen Intention handelt es sich in allen Fällen um eher privilegierte Familien, in denen beide Elternteile zeitliche und finanzielle Ressourcen in die Kompensation der pandemiebedingten Einschränkungen investierten und daneben auch die zeitlichen und sozialen Ressourcen aufbrachten, um sich an unserer Studie zu beteiligen. Dennoch ist es gelungen, eine gewisse Bandbreite an Wohnsituationen (in Bezug auf Gebäudetyp, zur Familie gehörige Personen, Betreuungssituation) und auch an Freiraumzugängen (eigener Garten oder Gemeinschaftsgarten, Ausstattung, Bewegungsradius) abzubilden. Die Auswirkungen auf Kinder und Familien mit weniger Kompensationsressourcen und weniger Zugang zum wohnungsnahen Freiraum sind vor diesem Hintergrund vorstellbar. Dennoch bedarf es hier weiterer Studien mit höheren Fallzahlen und einer breiteren Beteiligung.

Neben der Sondersituation der Pandemie wurden die Ergebnisse aber auch durch Faktoren der Interviewsituation beeinflusst. Es ist auffällig, dass bei keinem Interview Karten mit räumlichen Verknüpfungen einzelner Situationen, mit Wegen und Verbindungen entstanden sind, sondern fast ausschließlich einzelne Bilder, die auf dem Zeichenblatt nebeneinanderstehen. Im Gespräch erläuterten die Kinder durchaus räumliche Zusammenhänge, stellten diese jedoch nicht zeichnerisch dar. Hier stellt sich die Frage nach den Gründen. Hängt es mit der Zeitspanne zusammen, die zwischen dem Betrachtungszeitraum und dem Zeitpunkt des Interviews lag, durch dieviele Einzelheiten in Vergessenheit gerieten? Liegt es an der Formulierung der Aufgabezu Beginn des Interviews oder am Alter und Entwicklungsstand der Kinder, dass es schwierigfür siewar, dieseräumlichen Zusammenhängedarzustellen? Eine inhaltliche Interpretation ist gleichfalls möglich, schließlich legten die Kinder viel weniger Wege zurück. Die (eher langen) Wege, die beispielsweise bei Ausflügen zurückgelegt wurden, sind wiederum sehr anspruchsvoll zu zeichnen und würden auch Erwachsene überfordern. Dazu passt die bereits 1976 von Stephen Grabow und Neil J. Salkind gemachte Beobachtung, dass Kinder diejenigen Räume und Orte, denen sie selbst die meiste Bedeutung zuweisen, auch deutlich differenzierter und facettenreicher zeichnen (Grabow/Salkind 1976).

Mehrere Kinder kommentierten während desZeichenprozessesihr eigenes Zeichenvermögen. So wurdeverbalisiert, wo sie Probleme hatten oder hinter der eigenen Erwartung zurückblieben: „Ja, ich mache erst mal den Eimer. Blätter kann ich nicht malen “(K1), „Ist hier irgendwienicht so gut geworden“ (K6) oder „Sieht jetzt nicht aus wie ein Trampolin“(K4). Hier zeigt sich eine grundsätzliche Herausforderung dieser Methode, die bei Erwachsenen und Kindern ab einem gewissen Alter zutrifft. Je stärker die Kinder reflektieren konnten, dass ihre Zeichnung sich nicht mit ihrem Bild von der Wirklichkeit deckte, desto schneller waren sieunzufrieden mitihrerZeichnung. Das älteste, 
stark digital affine Kind löste das Problem durch eine eher piktogrammartige „Karte“. So ist zu vermuten, dass bei älteren Kindern das Abstraktionsvermögen zunimmt und dadurch ihre Ansprüche an ein „schönes Bild“ sinken.

Auch dieAnwesenheit der Eltern hat so manche Interviewsituation beeinflusst. Während es einigen Eltern sehr gut gelang, während des Interviews mit ihrem Kind selbst im Hintergrund zu bleiben, gaben andere Erinnerungshilfen und konnten es teilweise schlecht aushalten, wenn das Kind sich bei den Zeichnungen Zeit ließ oder wenig erzählte. Sie kommentierten auch den Zeichenprozess: „Verliere dich nicht in Details, okay? Willst du mal was erzählen dazu? [...] Ich glaube, die Blumenkästen sind jetzt nicht so wichtig, die Blumenkästen von den Nachbarn, oder?“ (E2) oder „Ja, aber, [Kind], darum geht es nicht. Es geht um den Hinterhof" (E4). Hier scheint es wichtig zu sein, die Eltern noch deutlicher im Hinblick auf ihre zunächst beobachtende Rolle zu instruieren und sie darauf hinzuweisen, dass ein solches, scheinbar nicht zielgerichtetes Vorgehen ganz normal ist und durchaus auch über Umwege zu nutzbaren Daten führen kann.

Darüber hinaus hatten die räumlichen Rahmenbedingungen des Interviews einen Einfluss auf dessen Verlauf. Bei einigen Interviews konnten wir beobachten, dass das Kind den Raum, wenn er in Sichtweite war, als Erinnerungsstütze verwendete. Andererseits kam es auch vor, dass ein Kind dann vermehrt das zeichnete, was es sah, und nicht, an was es sich erinnerte. Daran konnten wir ablesen, dass die Situation des Zeichnens und des Interviews insgesamt auch bei den Kindern etwas auslöste. Einem Kind fiel es zum Beispiel schwer, sich von seinen Zeichnungen zu trennen und wir mussten ihm versprechen, sienach der Auswertung zurückzugeben. Bei einemanderen Kind kam es dazu, dass es durch das Zurückerinnern an die Frühphase der Pandemie ein Spiel wieder aufgriff, das es im Frühling oft gespielt hatte. Es ist dementsprechend wichtig zu reflektieren, dass (qualitative) Forschung immer auch Einfluss auf den Alltag des Forschungssubjekts nimmt.

\section{Kinder als Alltagsexpert_innen ernst nehmen: Ausblick}

Wie eingangs geschildert, fordern Studien eine „Post-Corona-Stadt“, „NahErholungsmöglichkeiten” zu stärken und die „Bedeutung von privat verfügbarem Freiraum und die damit verbundenen sozialen Privilegierungen" zu reflektieren (Schneidewind et al. 2020: 7). Diese Forderungen erhalten mit Hinblick auf die Freiraumpraktiken von Kindern und Familien nach Auswertung unserer Ergebnisse weiteres Gewicht. Wir konnten zeigen, dass die wohnungsnahen Freiräume eine große Bedeutung für Kinder haben und mit ihnen ein beträchtlicher Teil der Kompensationsstrategien zusammenhängt, die genutzt wurden, um mit den Einschränkungen des Familienalltags umzugehen. Es muss die Frage gestellt werden, welche Lerneffekte für die Freiraumplanungund-gestaltungaus dieser Sondersituation entstehen können. Was kann daraus für die Freiraumpraktiken im Familienalltag nach Corona abgeleitet werden? Welche Erkenntnisse sind für zukünftige Planungsprozesse von Wohnung und Wohnumfeld relevant?

Freiräume, das (unbeaufsichtigte, nicht instutionalisierte) Bewegen und Spielen an der frischen Luft sowie die selbstständige Erreichbarkeit dieser Räume sind (neben Wohnräumen und familiärem Umfeld) für Kinder auch 
über Krisensituationen hinaus sehr wichtig. Sie fördern die körperliche Gesundheit und Kreativität und bieten Ausweichmöglichkeiten, wenn die Stimmung in der Familie angespannt ist. Sie ermöglichen selbstständige, teilweise unbeaufsichtigte Spiel- und Bewegungserfahrungen, in denen die Kinder Motorik und sicheres Bewegen außerhalb der eigenen Wohnung trainieren. Gerade in Zeiten der Coronapandemie - so zeigen unsere Ergebnisse eindrücklich - waren sie für manche der einzige Ort, an dem reale Kontaktemit anderen Kindern noch möglich waren, da dieAnsteckungsgefahran der frischen Luft als gering galt. Nicht zuletzt hat sich gezeigt, dass gemeinschaftlich genutzte oder öffentliche wohnungsnahe Freiräume dazu beitragen können, elterliches Engagement in Teilen auszugleichen. Hier werden auch denjenigen Kindern die genannten Erfahrungen ermöglicht, deren Eltern nicht über ausreichende zeitliche und finanzielle Ressourcen verfügen, um regelmäßig Ausflüge zu machen oder die privaten Freiräume abwechslungsreich auszustatten. Gleichzeitig ist die Belastung für die Eltern, die diese Kompensationsleistungen erbringen mussten, nicht zu unterschätzen. Auch hier lassen sich die Langzeitfolgen noch nicht absehen. Daher ist das Thema kindergerechte Freiraumentwicklung eng verbunden mit der Förderung einer selbstständigen Mobilität von Kindern, welche in Deutschland und europaweit seit Jahren gesunken ist (Fyhri et al. 2011; Shaw et al. 2015).

Eine wichtige Schlussfolgerung lautet daher, dass die hohe Bedeutung des wohnungsnahen Freiraums sich lokal in sicherer Erreichbarkeit, angemessener Größe und Ausstattung widerspiegeln muss. Weiterhin sollte ein entsprechendes Augenmerk auf die Versorgung benachteiligter Quartiere gelegt werden. Darüber hinausist es wichtig, dass die planenden Disziplinen die Sicht der Kinder und Jugendlichen als Expert_innen ihres Alltags auch in Pandemiezeiten ernst nehmen, sie zur Sprache kommen lassen und ihre Praktiken und Bedürfnisse in Untersuchungen berücksichtigen.

Wir sehen unsere Studie als einen Anfang an, um diesen Fragestellungen nachzugehen. Den weiteren Forschungsbedarf haben wir aufgezeigt. In künftigen Studien istes wichtigzu beachten, dass bisher kaum weniger privilegierte Kinder und Familien repräsentiert sind. Vor dem Hintergrund, dass weitere Pandemiesituationen in den kommenden J ahren nicht ausgeschlossen werden können, stellt sich die Frage, wie auch diese bislang „unsichtbar“ gebliebenen Kinder aus Familien mit weniger großen sozialen, finanziellen und zeitlichen Ressourcen gesehen, beteiligt sowie ihre spezifischen Bedürfnisse in die Freiraumplanung einbezogen werden können. Über eine breitere und differenzierte Beteiligungsstrategie in Befragungen und Beteiligungsprozessen könnte zunächst erhoben werden, welche Arten von Benachteiligung die privat, gemeinschaftlich oder öffentlich zugänglichen wohnungsnahen Freiräume ausgleichen müssten und auf welche Weise allen Kindern und Familien Kompensationsstrategien und zugängliche, gegebenenfalls auch temporäre Ausgleichsräume ermöglicht werden können. Kinder müssen als Expert_innen für das Matschen, Basteln und Toben in die Planungsprozesse der für sie bestimmten und ihre Familien entlastenden Räume eingebunden werden.

Die Veröffentlichung dieses Aufsatzes erfolgte mit Mitteln aus dem OpenAccess-Publikationsfonds der Universitätsbibliothek Kassel. 


\section{Autor_innen}

HenrietteBertram ist Kulturwissenschaftlerin. Aktuell forscht siezu gendersensibler Planung und der Vereinbarkeit von Beruf und Familie.

henriette.bertram@asl.uni-kassel.de

Stefanie Hennecke ist Professorin für Freiraumplanung. Sie forscht zu aktueller und historischer Planung, Nutzung und Aneignung von Stadtgrün.

hennecke@uni-kassel.de

Angela Million ist Professorin für Städtebau und Siedlungswesen. Sie forscht zum Raumwissen von Kindern und lernenden und lehrenden Stadträumen.

a.million@isr.tu-berlin.de

Johanna Niesen ist interdisziplinäre Stadt- und Freiraumforscherin. Ihre Forschungsinteressen sind Aneignungspraktiken und das Raumwissen von Kindern und Jugendlichen.

johanna.niesen@uni-kassel.de

\section{Literatur}

Andresen, Sabine / Lips, Anna / Möller, Renate / Rusack, Tanja / Schröer, Wolfgang / Thomas, Severine / Wilmes, Johanna (2020a): Kinder, Eltern und ihre Erfahrungen während der Corona-Pandemie. Erste Ergebnisse der bundesweiten Studie KiCo. Hildesheim: Universitätsverlag Hildesheim.

Andresen, Sabine / Lips, Anna / Rusack, Tanja / Schröer, Wolfgang / Thomas, Severine / Wilmes, Johanna (2020b): Nachteile von Kindern, Jugendlichen und jungen Erwachsenen ausgleichen. Politische Überlegungen im Anschluss an die Studien JuCo und KiCo. Hildesheim: Universitätsverlag Hildesheim.

Bähr, Sebastian / Frodermann, Corinna / Stegmaier, Jens / Teichler, Nils / Trappmann, Mark (2020): Knapper Wohnraum, weniger IT-Ausstattung, häufiger alleinstehend: Warum die Corona-Krise Menschen in der Grundsicherung hart trifft. Nürnberg: IAB-Forum.

Ball, David / Gill, Tim / Yates, Andy (2020): COVID-19 and children's play. Play Safety Forum.

Behnken, Imbke / Zinnecker, Jürgen (2010): Narrative Landkarten. Ein Verfahren zur Rekonstruktion aktueller und biografisch erinnerter Lebensräume. Weinheim: Juventa.

Bertelsmann-Stiftung (2020): Factsheet. Kinderarmut in Deutschland. Gütersloh.

BMBF - Bundesministerium für Bildung und Forschung (2020): Memorandum Post-CoronaStadt. Für eine suffiziente und resiliente Entwicklung von Städten und Regionen. https://www.nachhaltige-zukunftsstadt.de/downloads/20200729_Memorandum_ Post-Corona-FINAL_BMBF.pdf (letzter Zugriff am 10.12.2020).

Burke, Catherine (2005): „Play in focus”. Children researching their own spaces and places for play. In: Children, Youth and Environments 15/1, 27-53.

Burke, Kevin J. / Greene, Stuart / McKenna, Maria K. (2016): A critical geographic approach to youth civic engagement. In: Urban Education 51/2, 143-169.

Chawla, Louise (2016): Growing up in an urbanizing world. Florence: Taylor and Francis.

Claßen, Thomas (2020): Gesundheitsförderliche Stadtentwicklung. Zwischen Renaissance und Neuerfindung. In: Informationen zur Raumentwicklung 1/2020, 4-17.

Curtis, Jacqueline W. / Shiau, Ellen / Lowery, Bryce / Sloane, David / Hennigan, Karen / Curtis, Andrew (2014): The prospects and problems of integrating sketch maps with geographic information systems to understand environmental perception. A case study of mapping youth fear in Los Angeles gang neighborhoods. In: Environment and Planning B: Urban Analytics and City Science 41/2, 251-271.

DAKJ - Deutsche Akademie für Kinder- und Jugendmedizin e. V.(2020): Stellungnahme der Deutschen Akademie für Kinder- und Jugendmedizin e. V. zu weiteren Einschränkungen der Lebensbedingungen von Kindern und J ugendlichen in der Pandemie mit dem neuen Coronavirus (SARS-CoV-2). https://www.dakj.de/stellungnahmen/stellungnahmeder-deutschen-akademie-fuer-kinder-und-jugendmedizin-e-v-zu-weitereneinschraenkungen-der-lebensbedingungen-von-kindern-und-jugendlichen-in-derpandemie-mit-dem-neuen-coronavirus-sar/ (letzter Zugriff am 10.12.2020). 
Fischer, Natalie / Heinzel, Friederike / Lipowsky, Frank / Züchner, Ivo (2020): Kinder und Jugendliche in der Corona-Krise: Herausforderungen und mögliche Ansätze für pädagogisches und politisches Handeln. Arbeitspapier: https://www.uni-kassel.de/ fbo1/index.php? $\mathrm{eID}=$ dumpFile\&t $=\mathrm{f} \& \mathrm{f}=427 \&$ token $=43221$ bce $47504 \mathrm{aa} 658376 \mathrm{f} 7 \mathrm{adbo} 184$ 8829b52517 (letzter Zugriff am 7.6.2021).

Fölling-Albers, Maria (2000): Entscholarisierung von Schule und Scholarisierung von Freizeit? Überlegungen zu Formen der Entgrenzung von Schule und Kindheit. In: ZSE Zeitschrift für Soziologie der Erziehung und Sozialisation 20/2, 118-131.

Fyhri, Aslak / Hjorthol, Randi / Mackett, Roger L. / Nordgaard Fotel, Trine / Kyttä, Marketta (2011): Children's active travel and independent mobility in four countries. Development, social contributing trends and measures. In: Transport Policy 18/5, 703-710.

Gebhard, Ulrich (2013): Kind und Natur. Die Bedeutung der Natur für die psychische Entwicklung. Wiesbaden: Springer Fachmedien.

Geis-Thöne, Wido (2020): Häusliches Umfeld in der Krise. Ein Teil der Kinder braucht mehr Unterstützung. Ergebnisse einer Auswertung des Sozio-oekonomischen Panels (SOEP). IW-Report 15/2020. Köln.

Graber, Kelsey M. / Byrne, Elizabeth M. / Goodacre, Emily J. / Kirby, Natalie / Kulkarni, Krishna / O'Farrelly, Christine / Ramchandani, Paul G. (2020): A rapid review of the impact of quarantine and restricted environments on children's play and health outcomes. Cambridge: University of Cambridge. https://psyarxiv.com/p6qxt/ (letzter Abruf am 5.10.2021).

Grabow, Stephen / Salkind, Neil J. (1976): The hidden structure of children's play in an urban environment. In: Peter Suedfeld / James A. Russel (Hg.), The behavioral basis of design. Beyond the applicability gap, Book 1. Proceedings, EDRA 7. Stroudsburg, PA: Dowden, Hutchinson and Ross, 164-171.

Honey-Rosés, Jordi / Anguelovski, Isabelle / Chireh, Vincent K. / Daher, Carolyn / van den Konijnendijk Bosch, Cecil / Litt, Jill S. / Mawani, Vrushti / McCall, Michael K. / Orellana, Arturo / Oscilowicz, Emilia / Sánchez, Ulises / Senbel, Maged / Tan, Xueqi / Villagomez, Erick / Zapata, Oscar / Nieuwenhuijsen, Mark J. (2020): The impact of COVID-19 on public space. An early review of the emerging questions - design, perceptions and inequities. In: Cities \& Health, 1-17.

Kampmann, Jan (2004): Societalization of childhood. New opportunities? New demands? In: Helene Brembeck / Barbro Johansson / Jan Kampmann (Hg.), Beyond the competent child. Roskilde: Roskilde University Press, 127-152.

Katz, Cindi (2004): Growing up global. Economic restructuring and children's everyday lives. Minneapolis: University of Minnesota Press.

Langmeyer, Alexandra / Guglhör-Rudan, Angelika / Naab, Thorsten / Urlen, Marc / Winklhofer, Ursula (2020): Kindsein in Zeiten von Corona. Erste Ergebnisse zum veränderten Alltag und zum Wohlbefinden von Kindern. München: Deutsches Jugendinstitut.

Lochner, Barbara (2020): Thüringer Familien in Zeiten von Corona - Wohlbefinden der Kinder, Herausforderungen des Homeschooling \& Unterstützungsbedarfe der Eltern. Erste Befunde. Erfurt: Fachhochschule Erfurt.

Million, Angela (2021a): Mental Maps und narrative Landkarten. In: Anna J. Heinrich / Séverine Marguin / Angela Million / Jörg Stollmann (Hg.), Handbuch qualitative und visuelle Methoden der Raumforschung. Bielefeld: transcript, 293-308.

Million, Angela (2021b): „No one listens to us...”. COVID-19 and its socio-spatial impact on children and young people in Germany. In: Children's Geographies, 2.4.2021, 1-9. https:// doi.org/10.1080/14733285.2021.1908520 (letzter Zugriff am 1.9.2021).

Moore, Sarah A. / Faulkner, Guy / Rhodes, Ryan E. / Brussoni, Mariana / Chulak-Bozzer, Tala / Ferguson, Leah J. / Mitra, Raktim / O’Reilly, Norm / Spence, John C. / Vanderloo, Leigh M. / Tremblay, Mark S. (2020): Impact of the COVID-19 virus outbreak on movement and play behaviours of Canadian children and youth. A national survey. In: The International Journal of Behavioral Nutrition and Physical Activity 17/1, 85 .

Moran, Mika R. / Plaut, Pnina / Merom, Dafna (2017): Is the grass always greener in suburban neighborhoods? Outdoors play in suburban and inner-city neighborhoods. In: International Journal of Environmental Research and Public Health 14/7, 759.

Razani, Nooshin / Radhakrishna, Rohan / Chan, Curtis (2020): Public lands are essential to public health during a pandemic. In: Pediatrics 146/2. https://doi.org/10.1542/ peds.2020-1271 (letzter Zugriff am 5.10.2021).

Schmitt, Gisela / Sommer, Ulrike / Wiechert, Carolin (2014): Wohnungsnahe Freiräume - Nutzerbezogene Raumqualitäten. Aachen: Rheinisch-Westfälische Technische Hochschule Aachen. 
Schneidewind, Uwe / Baedeker, Carolin / Bierwirth, Anja / Caplan, Anne / Haake, Hans (2020): „Näher“ - „Öffentlicher“ - „Agiler“. Eckpfeiler einer resilienten „Post-CoronaStadt“. Zukunftsimpuls Nr. 14. Wuppertal: Wuppertal Institut.

Schröer, Wolfgang (2021): Kindheit und Jugend in Zeiten von Corona. Konsequenzen für die aktuelle und zukünftige Kinder- und Jugendpolitik. Stellungnahme des Bundesjugendkuratoriums. München: Deutsches Jugendinstitut.

von Seggern, Hille (2009): Stadtsurfer, Quartierfans \& Co. Stadtkonstruktionen Jugendlicher und das Netz urbaner öffentlicher Räume. Berlin: Jovis.

Shaw, Ben / Bicket, Martha / Elliott, Bridget / Fagan-Watson, Ben / Mocca, Elisabetta / Hillman, Mayer (2015): Children's independent mobility. An international comparison and recommendations for action. London: Policy Studies Institute. http://www.psi.org. uk/children_mobility (letzter Zugriff am 1.9.2021).

Slater, Sandy J. / Christiana, Richard W. / Gustat, Jeanette (2020): Recommendations for keeping parks and green space accessible for mental and physical health during COVID-19 and other pandemics. In: Preventing Chronic Disease 17, E59.

Spitthöver, Maria (2002): Geschichte der Freiräume im Mietgeschosswohnungsbau. Kassel: Gesamthochschule Kassel.

Walper, Sabine / Reim, Julia / Schunke, Annika / Berngruber, Anne / Alt, Philipp (2021): Die Situation Jugendlicher in der Corona-Krise. München: Deutsches Jugendinstitut.

\section{Outdoor play during the Corona crisis. Children's and families' changing every-day geographies during the early weeks of the pandemic}

During the early weeks of the pandemic in Europe (mid-March to May), childcare facilities as well as public playgrounds were closed along with other institutions of public life. Even though the restrictions were not as drastic in Germany as elsewhere, many taken for granted outdoor activities became impossible from one day to the next. Thus, lockdown posed a severechallengeto families, incisively changing their routines and everyday geographies as well as the dynamics of public space. In our contribution, we will explore the pandemic-related restrictions in the everyday life of children and families and their compensation strategies. Using the method of narrative maps, we have analyzed the everyday geographies and daily routines of 8 to 12 year old children during lockdown in two German cities. We put forward the hypothesis that the use and appropriation of close tohome and private outdoor space such as backyards, balconies, sidewalks and allotment gardens increased during lockdown to compensate for the inaccessibility of other outdoor spaces. 